\title{
Das Problem der Dominanz bei Zwei-Personen-Spielen
}

\author{
J. NITSCHE
}

Eingegangen am 16. Oktober 1965

1. Im folgenden werden endliche Zwei-Personen-Spiele betrachtet, die sich durch Vorgabe der Strategienräume

$$
\begin{array}{ll}
X=\left\{x \mid x_{i} \geqq 0,\right. & \left.\sum_{1}^{m} x_{i}=1\right\} \\
Y=\left\{y \mid y_{k} \geqq 0,\right. & \left.\sum_{1}^{n} y_{k}=1\right\}
\end{array}
$$

und zweier Matrizen $A, B$ der Dimension $m \times n$ beschreiben lassen. Die Gewinne der zwei Spieler berechnen sich gemäß

$$
\varepsilon_{\mathrm{I}}=\varepsilon_{\mathrm{I}}(x, y)=x A y ; \quad \varepsilon_{\mathrm{II}}=\varepsilon_{\mathrm{II}}(x, y)=x B y .
$$

Handelt es sich um ein nicht-kooperatives Spiel und ist ein Gewinnaustausch zwischen den Spielern ausgeschlossen, so hat die Frage nach dominanten Strategien eine besondere Bedeutung.

Dabei heißt ein Strategienpaar $(x, y) \in X \times Y$ dominant, wenn kein anderes $\operatorname{Paar}(\bar{x}, \bar{y}) \in X \times Y$ mit

$$
\begin{aligned}
& \varepsilon_{\mathrm{I}}(\bar{x}, \bar{y}) \geqq \varepsilon_{\mathbf{I}}(x, y) \\
& \varepsilon_{\mathrm{II}}(\bar{x}, \bar{y}) \geqq \varepsilon_{\mathrm{II}}(x, y)
\end{aligned}
$$

existiert, wobei in mindestens einer der Ungleichungen das >-Zeichen gilt.

Unterstellt man nämlich ein ,rationales“ Verhalten beider Spieler, so werden diese sicher keine nicht-dominanten Strategien verwenden. Durch Utbergang zu diesen dominierenden Strategien würde sich der Gewinn für keinen der Spieler verschlechtern und für mindestens einen verbessern. Demgemäß kann unterstellt werden, daß dann beide gemäß stiller Übereinkunft die dominanten Strategien anwenden.

Hier soll die Frage untersucht werden, welche Punkte des Strategienraumes $X \times Y$ dominant sein können. Bei einem 0-Summen-Spiel oder einem damit strategisch äquivalenten ist offensichtlich jeder Punkt dominant; dieser Fall kann somit außer Betracht bleiben. - Das allgemeine Nicht-0-Summen-Spiel scheint von dieser Seite her bislang nicht betrachtet worden zu sein. NASH betrachtet in [3] dominante Strategien für einzelne Spieler, es wird also etwa ein $\bar{x} \in X$ mit $\varepsilon_{\mathrm{I}}(\bar{x}, y)>\varepsilon_{\mathrm{I}}(x, y)$ für alle $(x, y) \in X \times Y$ gesucht. Solche Strategien $\bar{x}$ müssen am Rande von $X$ liegen. Ein Satz von KreuLE (Satz 2 in [2]), wonach ein eindeutiges inneres Gleichgewichtsstrategienpaar sicher nicht dominant ist, legt ebenfalls die Vermutung nahe, daß auch dominante Strategienpaare am Rande von $X \times Y$ liegen müßten. Wie sich jedoch zeigt, ist diese Aussage nur mit einer Aus- 
nahme richtig. Indem wir den Beweis bis 2. zurückstellen, sei dieser Ausnahmefall beschrieben:

Satz. $x$, y seien innere Punkte von $X$ bzw. Y. Soll ein einem 0 -Summen-Spiel nicht äquivalentes Spiel existieren, bei welchem $(x, y)$ dominant sind, so müssen die $M a-$ trizen $A, B$ von der Gestalt 1

$$
\begin{aligned}
& A=\varrho\left(a b-\varepsilon a e^{\prime}-\varepsilon e b+\varepsilon^{2} e e^{\prime}\right)+a e^{\prime}+e b-\varepsilon e e^{\prime} \\
& B=\sigma\left(a b-\varepsilon a e^{\prime}-\varepsilon e b+\varepsilon^{2} e e^{\prime}\right)-\lambda\left(a e^{\prime}+e b\right)+\nu e e^{\prime}
\end{aligned}
$$

sein. Dabei gilt

i) $x a=b y=\varepsilon$

ii) $\lambda \geqq 0$

iii) $\varrho \sigma>0$.

Setzt man nämlich für zwei andere Strategien $\bar{x}, \bar{y}$ :

$$
\bar{x}=x+u, \quad \bar{y}=y+v,
$$

so ergibt sich wegen

$$
u e=e^{\prime} v=0
$$

mit den Abkürzungen

$$
\xi=u a, \quad \eta=b v
$$

für die Gewinne

$$
\begin{aligned}
& \varepsilon_{\mathrm{I}}(\bar{x}, \bar{y})-\varepsilon_{\mathrm{I}}(x, y)=\varrho \xi \eta+\xi+\eta \\
& \varepsilon_{\mathrm{II}}(\bar{x}, \bar{y})-\varepsilon_{\mathrm{II}}(x, y)=\sigma \xi \eta-\lambda(\xi+\eta) .
\end{aligned}
$$

Der Raum $X \times Y$ projiziert sich auf ein Rechteck

$$
\stackrel{\circ}{X}=\{\xi \mid \underline{\xi} \leqq \xi \leqq \bar{\xi}\}, \quad \stackrel{\circ}{Y}=\{\eta \mid \underline{\eta} \leqq \eta \leqq \bar{\eta}\}
$$

mit

$$
\underline{\xi}=\underset{\bar{x} \in X}{\operatorname{Min}}(\bar{x}-x) a
$$

und ähnlichen Ausdrücken für die übrigen Schranken.

Die Flächen gleichen Gewinns für die Spieler sind in der $\xi-\eta$ Projektion Hyperbeln, die sich im Nullpunkt $(\bar{x}=x, \bar{y}=y$ ) berühren. Daraus ergibt sich unmittelbar geometrisch, daß außer den Eckpunkten weiterhin auch sämtliche Punkte der Winkelhalbierenden $\xi=\eta$ dominant sein können.

Je nach den Werten von $\underline{\xi}, \bar{\xi}, \underline{\eta}, \bar{\eta}$ einerseits und $\varrho, \sigma, \lambda$ andererseits sind die Fälle zu unterscheiden:

a) Alle Punkte der Winkelhalbierenden sind Dominanzpunkte;

b) ein innerer Teil der Winkelhalbierenden $\left(\xi=\eta\right.$ mit $\left.|\xi| \leqq \xi_{0}\right)$ trägt dominante Punkte. Dazu kommen die Punkte $(\bar{\xi}, \bar{\eta})$ bzw. $(\underline{\xi}, \underline{\eta})$ und gegebenenfalls Teile des Randes;

c) nur die Randpunkte rechts oben bzw. links unten sind dominant.

1 Hierbei bedeuten $a, e$ bzw. $b, e^{\prime}$ jeweils $m$-dimensionale Spalten- bzw. $n$-dimensionale Zeilen-Vektoren; die Komponenten von $e$ rzw. $e^{\prime}$ sind sämtlich gleich eins. 
Für ein solches Spiel findet man als einzigen Gleichgewichtspunkt im Sinne von NASH (bei $\lambda \sigma>0$ )

$$
\xi=\operatorname{Min}(\bar{\xi}, \lambda / \sigma) ; \eta=\operatorname{Max}(\underline{\eta},-1 / \varrho)
$$

der in Übereinstimmung mit dem erwähnten Resultat von KRELLw nicht dominant ist.

2. Zur bequemeren Schreibweise werden wir im folgenden zwei Vektoren $a$ und $b$ als kongruent modulo einem linearen Teilraum $\&$ bezeichnen, falls der Differenzvektor $a-b$ in $\mathfrak{R}$ enthalten ist:

Definition. $a \equiv b(\mathfrak{\Omega}) \Leftrightarrow a-b \in \mathfrak{\Omega}$.

Weiterhin sollen $U, V$ Umgebungen des Nullpunktes (aufgefaßt als Punkt des $m$ - bzw. des $n$-dimensionalen Raumes) in den Hyperebenen $u e=0 \mathrm{bzw} \cdot e^{\prime} v=0$ bedeuten:

Definition.

$$
\begin{aligned}
& U=\left\{u|| u_{i} \mid<\delta \wedge u e=0\right\} \\
& V=\left\{v|| v_{j} \mid<\delta \wedge e^{\prime} v=0\right\} .
\end{aligned}
$$

Zum Beweis des Satzes wird das Lemma benötigt:

Lemma. Es sei \& ein linearer Teilraum und es gelte für das Matrizenpaar $A, B$ :

$$
B v \equiv-\lambda(v) A v(\Omega)
$$

für alle $v \in V$ mit einer Skalarfunktion $\lambda(v)$. Dann gilt entweder

$$
\lambda=\text { konst. }
$$

oder es ist

$$
A v \equiv 0(\Omega), \quad B v \equiv 0(\Omega) \quad \text { für } \quad v \in V .
$$

Beweis. Soll der zweite Fall nicht vorliegen, so muß für mindestens ein $v_{0} \in V$ gelten

$$
A v_{0} \notin \mathfrak{R} \text {. }
$$

Nun sei $p$ ein beliebiger zu $\mathfrak{\&}$ orthogonaler Vektor, der nicht gleichzeitig orthogonal zu $A v_{0}$ ist. Dann liefert die Multiplikation der Kongruenzbeziehung (5) mit $p$ :

$$
p B v=-\lambda p A v \text {. }
$$

Daraus folgt, daß $\lambda$ eine gebrochen lineare Funktion in $v$ ist. Wären nun die zu $e^{\prime}$ orthogonalen Anteile von $p B$ und $p A$ linear unabhängig, so lieBe sich ein $v_{1}$ mit $p B v_{1} \neq 0, p A v_{1}=0$ finden. Da andererseits wegen $v \in V$ die Anteile in Richtung $e^{\prime}$ herausfallen, folgt wie behauptet $\lambda=$ konst.

Nach diesen Vorbemerkungen wenden wir uns dem Beweis des Satzes zu. Wir unterstellen, daß $(x, y)$ ein im Innern von $X \times Y$ gelegenes dominantes Strategienpaar ist. Werden $U, V$ gemäß

$$
\begin{aligned}
& U=\left\{u|| u_{i} \mid<\operatorname{Min}\left(x_{i}, 1-x_{i}\right) \text { und } u e=0\right\} \\
& V=\left\{v|| v_{j} \mid<\operatorname{Min}\left(y_{j}, 1-y_{j}\right) \text { und } e^{\prime} v=0\right\}
\end{aligned}
$$

definiert, so gilt jedenfalls

$$
\begin{array}{lll}
\bar{x}=x+u \in X & \text { für } & u \in U \\
\bar{y}=y+v \in Y & \text { für } & v \in V .
\end{array}
$$


Aus der Definition der Dominanz ergibt sich bei Einsetzen von (7) in (3) somit:

$$
\begin{array}{ll}
\text { Aus } & u A y+x A v+u A v \geqq 0 \\
\text { folgt } & u B y+x B v+u B v \leqq 0
\end{array}
$$

und umgekehrt; d. h., ist der 2. Ausdruck nicht negativ, so muß der erste nicht positiv sein.

Diese zwei Beziehungen lassen sich als voneinander abhängige lineare Ungleichungen für $u$ bzw. $v$ auffassen. Daraus folgt (vgl. [1], S. 103)

$$
\begin{aligned}
B y+B v & =-\lambda(A y+A v)+\varrho e \\
x B v & \leqq-\lambda x A v
\end{aligned}
$$

und

$$
\begin{aligned}
x B+u B & =-\mu(x A+u A)+\sigma e^{\prime} \\
u B y & \leqq-\mu u A y .
\end{aligned}
$$

Hierbei sind $\lambda=\lambda(v)$ und $\mu=\mu(u)$ positiv, während $\varrho=\varrho(v), \sigma=\sigma(u)$ beliebiges Vorzeichen haben können.

In $\left(9_{1}\right) v=0$ eingesetzt gibt speziell

und damit

$$
B y=-\lambda_{0} A y+\varrho_{0} e
$$

$$
u B y=-\lambda_{0} u A y
$$

Somit kann aus $\left(10_{2}\right)$

$$
\left(\lambda_{0}-\mu(u)\right) u A y \geq 0
$$

gefolgert werden. Entsprechend ergibt sich

$$
\left(\mu_{0}-\lambda(v)\right) x A v \geq 0 .
$$

Also können zweimal zwei Fälle unterschieden werden:

$$
\begin{aligned}
& \text { 1. } u A y=0 \text { für } u \in U \\
& \text { 2. } \operatorname{sign}\left(\lambda_{0}-\mu(u)\right)=\operatorname{sign} u A y \\
& \text { 1'. } \quad x A v=0 \quad \text { für } \quad v \in V \\
& \left.2^{\prime} . \quad \operatorname{sign}\left(\mu_{0}-\lambda\right)(v)\right)=\operatorname{sign} x A V \text {. }
\end{aligned}
$$

Im Fall 1 ist

$$
A y \equiv 0(e)
$$

Gilt darüber hinaus

Fall 1a

$$
A v \equiv 0(e)
$$

so wäre

$$
A=e p+q e^{\prime}
$$

mit zwei Vektoren $p, q$. Wegen (13) und $y \in Y$, d. h. $e^{\prime} y=1$, müßte dann aber $q \equiv 0(e)$ gelten, so da $B$ sich (15) auf

$$
A=e p
$$


reduziert. Da nun (13) wegen (11) auch $B y \equiv 0(e)$ nach sich zieht und $\left(9_{1}\right)$ dann auch $B v \equiv 0(e)$ bedingt, gilt auch die Beziehung

$$
B=e \bar{p} .
$$

Dann liefert aber $\left(10_{1}\right)$ mit $u=0$

$$
\bar{p}=-\mu_{0} p+\sigma_{0} e
$$

bzw.

$$
B=-\mu_{0} A+\sigma_{0} e e^{\prime} .
$$

Das ist aber genau die Bedingung für die Aquivalenz mit einem Nullsummenspiel.

Im

Fall $1 b$

$$
\exists v_{0} \in V \quad \text { mit } A v_{0} \neq 0(e)
$$

kommen wir nach dem Lemma zu $\lambda=$ konst. Denn $\left(9_{1}\right)$ schreibt sich jetzt wegen (13) und (11)

$$
B v \equiv-\lambda A v(e)
$$

Demnach gilt

$$
B+\lambda A=e p+q e^{\prime} .
$$

Entsprechend wie oben ((15)-(18)) zeigt sich, daß dann $p$ und $q$ proportional zu $e^{\prime}$ und $e$ sind; d. h., es ergibt sich wieder ein einem Nullsummenspiel äquivalentes Spiel.

Es gilt nämlich jetzt

$$
\left(B+\lambda_{0} A\right) y=e(p y)+q,
$$

woraus wegen (11) $q \equiv 0(e)$ folgt. Außerdem ergibt sich aber mit $\left(9_{2}\right)$

$$
x\left(B+\lambda_{0} A\right) v=p v \leq 0,
$$

was $p v=0$ für $v \in V$ bzw. $p \equiv 0\left(e^{\prime}\right)$ nach sich zieht.

Da sich der Fall $\left.1^{\prime}\right)$ analog behandeln läßt, bleibt nur das Paar $\left(2,2^{\prime}\right)$ übrig. Hier scheiden die Annahmen

bzw.

$$
A v \neq 0(a y, e) \quad \text { für gewisse } v \in V
$$

$$
u A \neq 0\left(x a, e^{\prime}\right) \quad \text { für gewisse } u \in U
$$

sofort aus. Dann müBte nämlich nach dem Lemma $\lambda$ bzw. $\mu$ konstant sein, und wir kommen wieder zu (20), woraus analog die Äquivalenz mit einem Nullsummenspiel folgt.

Damit bleibt lediglich

$$
A v \equiv 0(A y, e) \quad u A \equiv 0\left(x A, e^{\prime}\right)
$$

übrig, woraus für $A$ die zwei Darstellungen

$$
\begin{aligned}
A & =A y p_{1}+e q_{1}+r_{1} e^{\prime} \\
& =p_{2} x A+e r_{2}+q_{2} e^{\prime}
\end{aligned}
$$


resultieren. Multiplikation mit $x$ und $y$ und Vergleich ermöglicht die Elimination der Vektoren $p, q, r$ und liefert nach einfacher Umformung

$$
A=\varrho\left(A y x A-\varepsilon A y e^{\prime}-\varepsilon e x A+\varepsilon^{2} e e^{\prime}\right)+A y e^{\prime}+e x A-\varepsilon e e^{\prime},
$$

wobei $\varepsilon=x A y$ gesetzt ist. Führt man noch $a=A y, b=x A$ ein, so ist die angegebene Darstellung $\left(4_{1}\right)$ gewonnen. Diejenige für $B$ ergibt sich jetzt entsprechend, wenn nur die aus (22) folgenden Beziehungen

$$
B v \equiv 0(a y, e) \quad u B \equiv 0\left(x A, e^{\prime}\right)
$$

berücksichtigt werden. Die Vorzeichenbedingungen ii) und iii) des Satzes ergeben sich entweder durch die Vorzeichen in (9) ff. bzw. durch nachträgliche Fallunterscheidung.

\section{Literatur}

[1] FAN, K.: On systems of linear inequalities. Linear inequalities and related systems. Ann. Math. Studies, Nr. 38, 99-156 (1956).

[2] Krelle, W., u. D. Coenen: Das nichtkooperative Nichtnullsummen-2-Personenspiel. Unternehmensforsch. Heft 2/3, 9 (1965).

[3] NASH, J. : Noncooperative games. Ann. of Math. II. Ser. 54, 286-295 (1951).

Institut f. angew. Mathematik

der Albert-Ludwigs Universität

78 Freiburg i. Br.

Hebelstraße 40 